\title{
The Comparative Influence of 2 and 4 Weeks Preoperative Antituberculosis Treatment on Spinal Tuberculosis Surgery: A Multicenter, Prospective, Randomized Clinical Trial
}

\author{
Jun Fan · Tinglong Lan · Kai Tang · Guirong Wang • Weijie Dong • \\ Dawei Li · Guangxuan Yan · Yuan Li · Guohua Lei · Jianlin Shan • \\ Qinglei Wang · Jianhua Ma · Hairong Huang · Shibing Qin
}

Received: April 12, 2021 / Accepted: May 18, 2021 / Published online: June 13, 2021

(C) The Author(s) 2021

\section{ABSTRACT}

Introduction: A trade-off between successful surgery and minimizing the operation delay for patients with spinal tuberculosis (TB) is a major consideration to determine the duration of preoperational anti-TB treatment (AAT). In this study, 2 and 4 weeks preoperative AAT durations were compared for their influence on the operation outcomes.

Method: A multicenter, prospective, randomized trial was conducted in four hospitals in

Jun Fan, Tinglong Lan, Kai Tang and Guirong Wang contributed equally to this study.

Supplementary Information The online version contains supplementary material available at https://doi. org/10.1007/s40121-021-00462-2.

J. Fan · T. Lan · K. Tang - W. Dong · G. Yan · Y. Li · G. Lei · S. Qin $(\bowtie)$

Department of Orthopedics, Beijing Key Laboratory for Drug Resistant Tuberculosis Research, Beijing Chest Hospital, Capital Medical University, Beijing Tuberculosis and Thoracic Tumor Institute, Beiguan St \#9, Beijing 101149, China

e-mail: qinshibing2019@163.com

G. Wang · H. Huang ( $($ )

National Clinical Laboratory On Tuberculosis, Beijing Key Laboratory for Drug Resistant Tuberculosis Research, Beijing Chest Hospital, Capital Medical University, Beijing Tuberculosis and Thoracic Tumor Institute, Beijing, China e-mail: huanghairong@tb123.org
China. New patients with spinal TB were recruited and randomly allocated to two groups ( 2 or 4 weeks' preoperative treatment) and administered the standardized first-line anti-TB drugs. The symptom changing and indicators reflecting recovery and side effects of the treatment were monitored. Patient was followed up for another 18 months after completion of treatment.

Results: In total, 150 eligible patients were enrolled between June 2014 and December 2016, and 13 patients were excluded after the enrollment. The remaining 137 participants were randomly allocated to the 2-week group $(n=68)$ or the 4 -week group $(n=69)$. These two groups acquired similar surgical outcomes, considering wound healing rate within 3 months after the operation $(94.20 \%, 65 / 69$ vs $89.71 \%, 61 / 68 ; P=0.333$ ) and bony fusion rate D. Li

The 8th Medical Center of Chinese PLA General Hospital, Beijing, China

J. Shan

Beijing Friendship Hospital, Capital Medical University, Beijing, China

Q. Wang · J. Ma

Beijing Geriatric Hospital, Beijing, China 
within 6 months $(98.46 \%, 64 / 65$ vs $95.45 \%$, $63 / 66 ; P=0.317)$. However, the culture positive rate of pus collected during operation in the 4-week group (41.94\%) was significantly lower than that of the 2-week group (60.94\%, $P=0.033)$. No reoccurrence of disease was observed in either group during the 18-month follow-up period.

Conclusion: Patients with spinal TB administered 2 or 4 weeks of preoperative anti-TB treatment acquired similar surgical outcomes. However, patients who underwent the operation sooner suffered 2 weeks less agony from the disease.

Keywords: Preoperative; Spinal tuberculosis; Therapy

\section{Key Summary Points}

Patients with spinal TB administered 2 or 4 weeks of preoperative anti-TB treatment acquired similar surgical outcomes.

The culture positive rate of pus collected during operation in the 4-week group (41.94\%) was significantly lower than the 2-week group (60.94\%, $P=0.033)$.

Only the location of the spinal lesion was significantly associated with non-healing wound or non-fusion bone.

Patients who underwent the operation sooner suffered 2 weeks less agony from the disease.

\section{DIGITAL FEATURES}

This article is published with digital features, including a summary slide, to facilitate understanding of the article. To view digital features for this article go to https://doi.org/10.6084/ m9.figshare.14605656.

\section{INTRODUCTION}

Tuberculosis (TB) is one of the most important infectious diseases and one of the most common causes of mortality in the world [1, 2]. Spinal tuberculosis, a type of extrapulmonary $\mathrm{TB}$, constitutes approximately 50\% of osteoarticular TB [3] and frequently causes neurologic deficit, kyphotic deformity, or even paraplegia. Although anti-TB chemotherapy is highly effective, surgery also play important roles in treating spinal $\mathrm{TB}$ and provides rapid relief, facilitating quicker eradication of the infection and preventing or treating neurologic deficit or spinal deformity [4]. Generally, preoperative anti-TB treatment (ATT) is administered to relieve symptoms in a timely manner. Case reports and small clinical series suggested that preoperative ATT might reduce the systemic and local bacterial toxins, alleviate symptoms, decrease leakage, and restrain abscesses [5-7]. On the hand, patients suffer enormously before the operation because of severe pain, limitation of movement, or even paraplegia, and usually require intensive care from others. Shortening the preoperative ATT is essential for patients not only because it could reduce suffering time but also because it could decrease the total medical care cost. However, currently there is no consensus on the appropriate duration of preoperative ATT for spinal TB treatment. Furthermore, no well-designed, prospective clinical trial has been performed for addressing this question. According to some retrospective studies on spinal TB surgical treatment, preoperative ATT duration times were applied arbitrarily, i.e., from 1 day to 3 months, and sometimes even longer [8-10]. The influence of preoperative ATT duration on the prognosis of bone TB is highly conflicting. Multiple studies found that preoperative ATT duration time did not cause differences in postoperative recurrence or nonhealing of spinal TB $[8,9]$, whereas Yang and Liu reported that inadequate preoperative ATT was an important risk factor of recurrence or non-healed spinal TB [10].

To elucidate the influence of the duration of preoperative chemotherapy on the outcome of surgical treatment for spinal $\mathrm{TB}$, we carried out a 
randomized, multicenter, prospective study in China to compare patients with different preoperative ATT duration time: 2 weeks and 4 weeks.

\section{METHODS}

\section{Ethical Approval}

The ethics approvals for this study were obtained from the Medical Research Ethics Committee, Beijing Chest Hospital, Capital Medical University (BJXK-20150901) and ethics approvals were also obtained for all study sites (Table 1 in the supplementary material). The study was conducted according to the Helsinki Declaration of 1964, as revised in 2013, concerning human and animal rights. Written informed consent was acquired from each participant.

\section{Study Design and Participants}

This multicenter, prospective, randomized trial was conducted in four hospitals in China (Beijing Chest Hospital, the 8th Medical Center of Chinese PLA General Hospital, Beijing Geriatric Hospital, and PLA Army General Hospital) between June 2014 and December 2016.

The enrolled patients with spinal TB were diagnosed according to the composite reference standard (CRS), which was composed of clinical findings, laboratory outcomes, histopathology reports, radiological imaging, and at least 18 months of follow-up after the date of enrollment. The inclusion criteria for this study were as follows: (1) new spinal TB case, defined as patient with spinal $\mathrm{TB}$ and had never been treated with anti-TB drug, (2) 15 years or older, (3) with definite surgical indications, (4) patient understood the nature of the study and gave informed consent. Exclusion criteria included (1) patient having concurrent active pulmonary $\mathrm{TB}$ and/or extrapulmonary TB other than spinal TB, (2) other concomitant illness that may interfere with surgery, (3) surgical contraindication, (4) psychiatric patient, (5) patient diagnosed with rifampicin and/or isoniazid resistance at any time by any method during the treatment episode.

Surgical indications included spinal instability, neurologic dysfunction, progression of kyphotic deformity, increased size of abscess and appearance of necrotic bone, progression of bone destruction, and neurologic deficit.

Patients were randomly $(1: 1)$ assigned to either a 2-week or a 4-week preoperative ATT group. Randomization was conducted by using a computer-generated random-number table, and a professional statistician generated the random allocation sequence. All participants and clinicians involved in this study were unblinded to the treatment allocation, while the radiologists were blinded. Patients received the standard chemotherapy including isoniazid (300 mg/day), rifampicin (450 mg/day), ethambutol (750 mg/day), and pyrazinamide $(1.5 \mathrm{~g} /$ day $)$ for 2 or 4 weeks before the operation. The trial was monitored by an independent data and safety monitoring committee. All patient information was routinely collected and recorded by the attending physicians.

\section{Treatment and Surgical Methods}

Simple debridement or debridement plus graft fusion was conducted depending on the patient's status. Posterior debridement was used for elderly patients or for cases with paraparesis. For others, anterior or anteroposterior debridement was performed on the basis of the location of the lesion. Laminectomy was also performed once there was oppression from the spinal cord's posterior column. Standard anti-TB treatment (6HREZ/12HRE) was administered postoperatively for another 18 months.

\section{Assessment of Progress}

\section{Patient Follow-Up}

Patients remained hospitalized from enrollment to the completion of postoperative care. Then patients were assessed longitudinally at 1-month intervals up to 3 months, and 3-month intervals up to 18 months, postoperatively. On each assessment, the following indexes were recorded: (1) blood indicators, 
such as erythrocyte sedimentation rate (ESR), and hepatorenal function; (2) wounds healing and sinus formation; (3) bone fusion; (4) internal fixation loosening or displacement and fracture; (5) visual analogue scale (VAS) score; and (6) postoperative complications. The primary endpoint was the surgical outcome including wound healing within 3 months and bone fusion within 6 months. Another 18 month's follow-up was conducted after completion of anti-TB therapy by telephone enquiry every 6 months.

\section{Debridement Specimen Examination}

A pus specimen collected during the operation from each patient was subjected to smear microscopy, culture, and Xpert MTB/RIF (Xpert) assay (Cepheid, Sunnyvale USA), while a tissue specimen was subjected to histopathology examination, simultaneously. Drug susceptibility testing (DST) was conducted for all the recovered isolates.

\section{Statistical Analysis}

SPSS version 19.0 (IBM, Armonk, NY, USA) was used to compare baseline clinical characteristics and the demographic data via Mann-Whitney $U$ test for continuous variables and chi-squared test for categorical variables. In addition, univariate analysis and multivariate analysis were conducted to assess the potential risk factors associated with a poor clinical outcome, respectively. The difference was declared as significant if the $P$ value was less than 0.05 .

\section{RESULTS}

\section{Patient Characteristics}

A total of 165 patients were screened and 150 underwent randomization in this study. The diagnosis of 130 patients was confirmed by either smear microscopy, culture, or Xpert with pus/tissue examinations, whereas for the remaining 20 patients, the diagnosis was referred to the pathology examination outcomes with tissues. During the study period, 13 patients discontinued trials, so that the final sample size for analysis was 137 (Fig. 1), which included 68 and 69 patients in 2- or 4-week groups, respectively. All of the patients were HIV-uninfected. The demographic and clinical characteristics of the patients were similar in the two study groups (Table 1 ).

\section{Preoperative Chemotherapy Efficacy}

\section{Wound Healing}

Overall $94.20 \%(65 / 69)$ of the patients healed well within 3 months after the operations in the 4-week preoperative ATT group, a rate which was slightly higher than that in the 2-week preoperative ATT group $(89.71 \%, 61 / 68 ; P=0$. $333)$. However, there was no significant difference. Seven $(10.2 \%, 7 / 68)$ patients had postoperative non-healing in the 2-week group, including three with thoracolumbar TB, two with thoracic TB, and two with lumbosacral TB. Four $(5.80 \%, 4 / 69)$ patients had postoperative non-healing in the 4 -week preoperative ATT group, including two with thoracolumbar TB and two with lumbosacral TB.

\section{Bone Fusion}

Debridement was performed for six cases; debridement and bone graft fusion internal fixation were performed for 131 cases in this study. Bony fusion rate within 6 months after operation in the 4-week group $(98.46 \%, 64 / 65)$ was slightly higher than that in the 2-week group $(95.45 \%, 63 / 66 ; P=0.317)$, although there was no significant difference between these two groups (Table 2). The fusion time was similar in the two groups $(3.23 \pm 0.77$ months vs $3.28 \pm 0.81$ months, $P=0.723$ ) (Table 2 , Fig. 2). Two patients had bone non-fusion outcome in the 2-week group, one with lumbar TB and the other with lumbosacral TB. In contrast, only one lumbosacral TB patient in the 4-week group had bone non-fusion outcome.

\section{ESR Normalization}

ESR normalization was relatively quicker in the 4-week group than in the 2-week group, but the difference was not significant (Table 3, Fig. 3). 


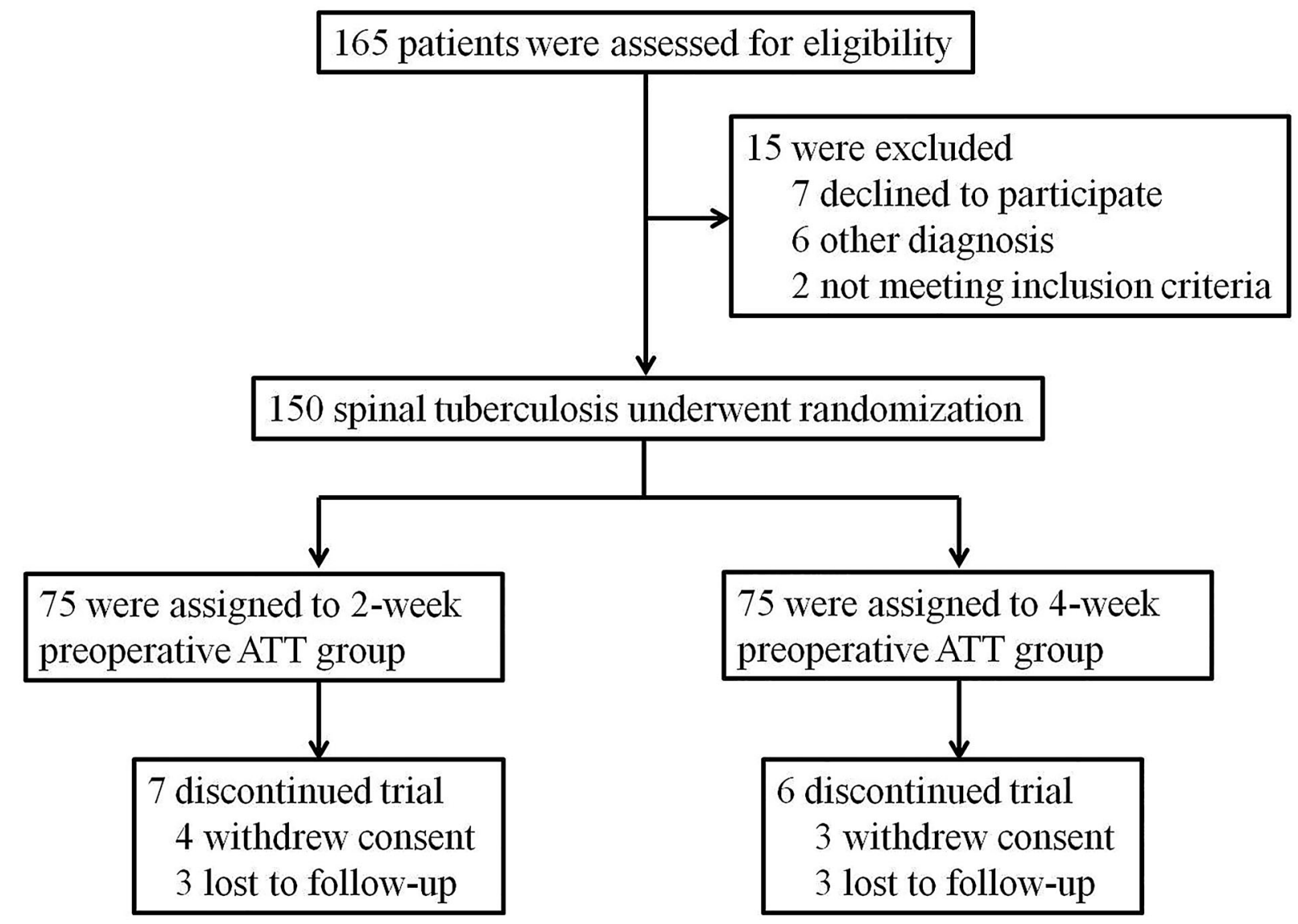

Fig. 1 Recruitment of the participants

\section{VAS Score Improvement}

There was significant pain relief after the surgery. The average VAS decreased from $4.79 \pm 1.62$ preoperatively to $2.23 \pm 1.31$ one month post-operation $(P<0.001)$ and then to $0.64 \pm 0.81$ at the final follow-up $(P<0.001)$ in the 2-week group, while the average VAS decreased from $4.35 \pm 2.36$ preoperatively to $2.35 \pm 1.48$ one month post-operation $(P<0.001)$ and then to $0.72 \pm 0.78$ at the final follow-up $(P<0.001)$ in the 4 -week group. The difference in VAS was not significant between the two groups either pre-operation or postoperation (Fig. 4).

\section{Examination Outcomes of the Specimen Collected During Operation}

The positive rate by Xpert with pus in the 4-week preoperative ATT group (86.21\%) was higher than that in the 2-week group $(80.00 \%$, $P=0.388$ ) with no significant difference. However, the positive rate of culture with pus in the 4-week group (41.94\%) was significantly lower than that in the 2-week group (60.94\%) $(P=0.033)$ (Table 4). In total, $88.32 \%(121 / 137)$ of the enrolled patients had bacteriological evidence of $\mathrm{TB}$, which indicates that at least one positive outcome was acquired by smear, culture, or Xpert assay. All the other patients had supportive $\mathrm{TB}$ diagnosis according to tissue pathology examinations.

\section{Factors Linked to Wound Non-healing or Bone Non-fusion}

A total of 13 patients $(9.49 \%, 13 / 137)$ presented unfavorable outcomes, including 11 non-healing wound and/or 3 non-fusion bone cases. Of those five had thoracolumbar TB, six had 
Table 1 Demographic and clinical characteristics of spinal tuberculosis patients enrolled in this study

\begin{tabular}{|c|c|c|c|}
\hline Characteristics & 2-week group $(n=75)$ & 4-week group $(n=75)$ & $P$ value \\
\hline Age, median (range), year & $41(16-73)$ & $41(18-76)$ & 0.249 \\
\hline Gender (male/female) & $39 / 36$ & $50 / 25$ & 0.067 \\
\hline BMI & $22.35 \pm 3.21$ & $22.31 \pm 3.84$ & 0.831 \\
\hline Preoperative VAS score & $4.79 \pm 1.62$ & $4.35 \pm 2.36$ & 0.440 \\
\hline Paraplegia & $11(14.67)$ & $4(5.33)$ & 0.057 \\
\hline Clinically evident abscess & $17(22.67)$ & $11(14.67)$ & 0.209 \\
\hline Limitation of movement & $26(34.67)$ & $30(40.00)$ & 0.500 \\
\hline Preoperative ESR (mm/h) & $40.94 \pm 30.82$ & $38.10 \pm 27.94$ & 0.744 \\
\hline \multicolumn{4}{|l|}{ Site of lesion } \\
\hline Thoracic & $26(34.67)$ & $33(44.00)$ & 0.242 \\
\hline Lumbar & $33(44.00)$ & $23(30.67)$ & 0.091 \\
\hline Thoracolumbar & $7(9.33)$ & $8(10.67)$ & 0.785 \\
\hline Lumbosacral & $9(12.00)$ & $11(14.67)$ & 0.631 \\
\hline \multicolumn{4}{|l|}{ Number of segments involved } \\
\hline 1 level & $3(4.00)$ & $0(0.00)$ & 0.080 \\
\hline 2 levels & $55(73.33)$ & $58(77.33)$ & 0.570 \\
\hline 3 levels & $9(12.00)$ & $12(16.00)$ & 0.480 \\
\hline$\geq 4$ levels & $8(10.67)$ & $5(6.67)$ & 0.384 \\
\hline \multicolumn{4}{|l|}{ Surgical approach } \\
\hline Anterior & $2(2.67)$ & $1(1.33)$ & 0.560 \\
\hline Posterior & $32(42.67)$ & $38(50.67)$ & 0.326 \\
\hline Posterior and anterior & $41(54.67)$ & $36(48.00)$ & 0.414 \\
\hline Operation time (min) & $229.47 \pm 60.88$ & $225.33 \pm 51.62$ & 0.674 \\
\hline Blood loss (ml) & $621.09 \pm 358.55$ & $625.15 \pm 313.53$ & 0.945 \\
\hline Bone graft fusion & $66(88.00)$ & $65(86.67)$ & 0.806 \\
\hline Decompression & $25(33.33)$ & $21(28.00)$ & 0.479 \\
\hline \multicolumn{4}{|l|}{ Imaging findings } \\
\hline Narrow of disc space & $71(94.67)$ & $74(98.67)$ & 0.172 \\
\hline Loss of vertebral height & $66(88.00)$ & $65(86.67)$ & 0.806 \\
\hline Defect of vertebral bone & $75(100.00)$ & $75(100.00)$ & - \\
\hline Occupying of intraspinal space & $47(62.67)$ & $48(64.00)$ & 0.865 \\
\hline Widening of paravertebral soft tissue opacity & $71(94.67)$ & $74(98.67)$ & 0.172 \\
\hline Psoas abscess shadows & $35(46.67)$ & $29(38.67)$ & 0.322 \\
\hline
\end{tabular}


Table 1 continued

\begin{tabular}{llll}
\hline Characteristics & 2-week group $(\boldsymbol{n}=\mathbf{7 5 )}$ & 4-week group $(\boldsymbol{n}=\mathbf{7 5})$ & $\boldsymbol{P}$ value \\
\hline Subcutaneous abscess shadows & $3(4.00)$ & $2(2.67)$ & 0.649 \\
Concomitant pulmonary TB & $6(8.00)$ & $7(9.33)$ & 0.772 \\
\hline
\end{tabular}

Table 2 Preoperative chemotherapy treatment outcomes in patients with spinal tuberculosis

\begin{tabular}{llll}
\hline Treatment outcome & 2-week group $(\boldsymbol{n}=\mathbf{6 8})$ & 4-week group $(\boldsymbol{n}=\mathbf{6 9})$ & $\boldsymbol{P}$ value \\
\hline Wound healing within 3 month after operation & $61 / 68(89.71)$ & $65 / 69(94.20)$ & 0.333 \\
Bone fusion within 6 month after operation & $63 / 66(95.45)$ & $64 / 65(98.46)$ & 0.317 \\
Time to bone fusion postoperative (months) & $3.23 \pm 0.77$ & $3.28 \pm 0.81$ & 0.723 \\
\hline
\end{tabular}

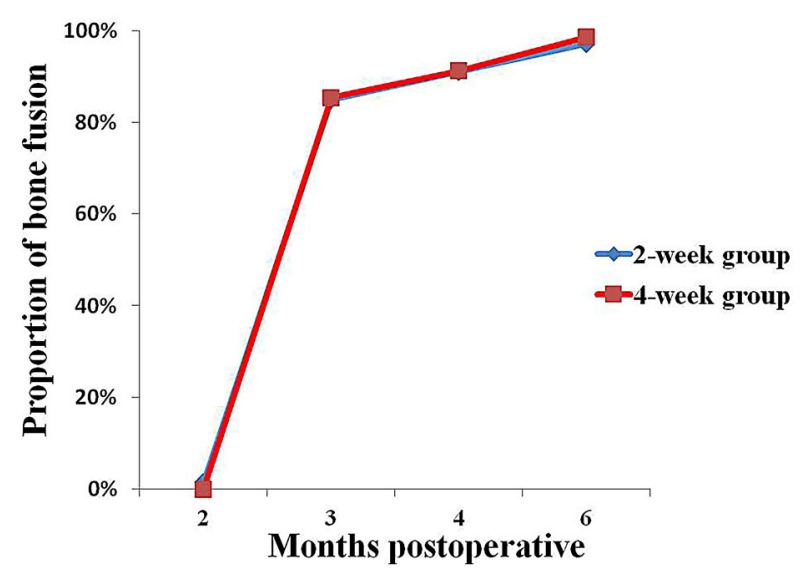

Fig. 2 Proportion of bone fusion postoperation

Table 3 Erythrocyte sedimentation recovery rate of the two groups

\begin{tabular}{lccc}
\hline ESR & 2-week group & 4-week group & P value \\
\hline Preoperative $(\mathrm{mm} / \mathrm{h})$ & $40.94 \pm 30.82$ & $38.10 \pm 27.94$ & 0.744 \\
1 month postoperative $(\mathrm{mm} / \mathrm{h})$ & $24.30 \pm 18.97$ & $20.86 \pm 17.04$ & 0.294 \\
6 months postoperative $(\mathrm{mm} / \mathrm{h})$ & $10.22 \pm 10.82$ & $7.81 \pm 12.88$ & 0.274 \\
9 months postoperative $(\mathrm{mm} / \mathrm{h})$ & $7.84 \pm 8.99$ & $5.59 \pm 5.80$ & 0.123 \\
Time to ESR normalization postoperative (months) & $3.40 \pm 3.33$ & $2.83 \pm 3.30$ & 0.315 \\
\hline
\end{tabular}




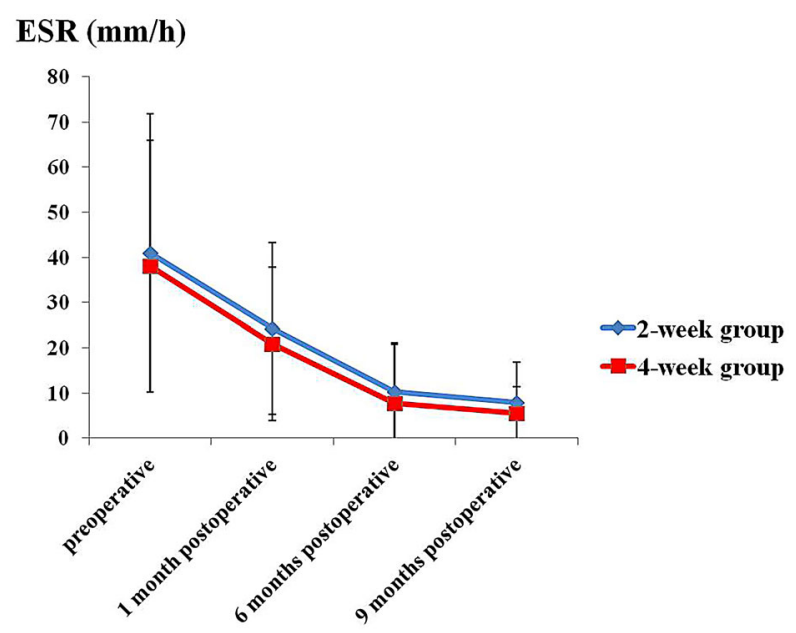

Fig. 3 Change of erythrocyte sedimentation rate (ESR) over time

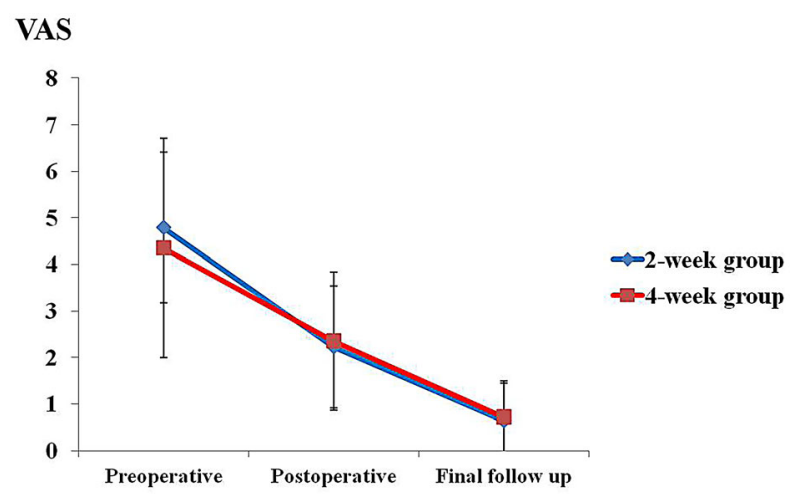

Fig. 4 Change of visual analogue scale (VAS) score over time

Table 4 Diagnostic accuracies of all methods compared to the composite reference standard

\begin{tabular}{llll}
\hline Methods & $\begin{array}{l}\text { 2-week group } \\
(\boldsymbol{n}=\mathbf{6 8})\end{array}$ & $\begin{array}{l}\text { 4-week group } \\
(\boldsymbol{n}=\mathbf{6 9})\end{array}$ & $\boldsymbol{P}$ value \\
\hline Smear & $15 / 62(24.19)$ & $15 / 62(24.19)$ & 1.000 \\
Culture & $39 / 64(60.94)$ & $26 / 62(41.94)$ & $\mathbf{0 . 0 3 3}$ \\
Xpert & $40 / 50(80.00)$ & $50 / 58(86.21)$ & 0.388 \\
\hline
\end{tabular}

lumbosacral TB, and two had thoracic TB. Logistic regression analysis indicated that age, sex, duration of preoperative ATT, preoperative ESR, pus TB culture results, the number of involved segments, surgical approach, operative duration, intraoperative blood loss, and concomitant pulmonary $\mathrm{TB}$ were not significantly associated with these unfavorable events (Table 5). Only the location of the spinal lesion was significantly associated with non-healing wound or non-fusion bone (Table 5). Lumbosacral vertebra TB had the highest chance of producing unfavorable outcome, followed by thoracolumbar vertebra and thoracic TB vertebra. Only one out of 53 patients with lumbar vertebra TB produced non-healing wound outcome. 
Table 5 Risk factors for wound non-healing or bone non-fusion in patients with spinal tuberculosis

\begin{tabular}{|c|c|c|c|c|c|c|}
\hline \multirow[t]{2}{*}{ Characteristics } & \multirow{2}{*}{$\begin{array}{l}\text { Wound healing } \\
\text { or bone fusion } \\
(n=124)\end{array}$} & \multirow{2}{*}{$\begin{array}{l}\text { Wound non- } \\
\text { healing or bone } \\
\text { non-fusion } \\
(n=13)\end{array}$} & \multicolumn{2}{|l|}{ Univariate analysis } & \multicolumn{2}{|c|}{ Multivariate analysis } \\
\hline & & & $\begin{array}{l}\text { Odds ratio }(95 \% \\
\text { CI) }\end{array}$ & $P$ value & $\begin{array}{l}\text { Odds ratio }(95 \% \\
\text { CI) }\end{array}$ & $P$ value \\
\hline $\begin{array}{l}\text { Age, median (range), } \\
\text { year }\end{array}$ & $44.52 \pm 16.83$ & $35.77 \pm 12.70$ & $\begin{array}{l}0.965 \\
\quad(0.928-1.004)\end{array}$ & 0.080 & & \\
\hline $\begin{array}{l}\text { Gender (male/ } \\
\text { female) }\end{array}$ & $72 / 52$ & $10 / 3$ & $\begin{array}{l}2.407 \\
\quad(0.631-9.181)\end{array}$ & 0.198 & & \\
\hline $\begin{array}{l}\text { Preoperative ATT } \\
\text { (2/4-week) }\end{array}$ & $60 / 64$ & $8 / 5$ & $\begin{array}{l}1.707 \\
\quad(0.529-5.507)\end{array}$ & 0.371 & $\begin{array}{l}0.725 \\
\quad(0.351-1.495)\end{array}$ & 0.383 \\
\hline $\begin{array}{l}\text { Preoperative ESR } \\
\qquad(\mathrm{mm} / \mathrm{h})\end{array}$ & $40.41 \pm 29.58$ & $35.42 \pm 27.01$ & $\begin{array}{l}0.994 \\
\quad(0.973-1.015)\end{array}$ & 0.573 & $\begin{array}{l}0.987 \\
\quad(0.960-1.014)\end{array}$ & 0.327 \\
\hline $\begin{array}{l}\text { Positive pus } \\
\text { Mycobacterium } \\
\text { tuberculosis } \\
\text { culture, } n \text { (\%) }\end{array}$ & $58(46.77)$ & $8(61.54)$ & $\begin{array}{l}1.931 \\
\quad(0.550-6.775)\end{array}$ & 0.304 & & \\
\hline Site of lesion & & & & 0.001 & & 0.007 \\
\hline Thoracic, $n(\%)$ & $52(41.94)$ & $2(15.38)$ & $\begin{array}{l}0.077 \\
\quad(0.014-0.429)\end{array}$ & 0.003 & $\begin{array}{l}0.088 \\
\quad(0.014-0.560)\end{array}$ & 0.010 \\
\hline Lumbar, $n(\%)$ & $51(41.13)$ & $1(7.69)$ & $\begin{array}{l}0.039 \\
\quad(0.004-0.357)\end{array}$ & 0.004 & $\begin{array}{l}0.049 \\
\quad(0.004-0.548)\end{array}$ & 0.014 \\
\hline $\begin{array}{l}\text { Thoracolumbar, } \\
n(\%)\end{array}$ & $9(7.26)$ & $4(30.77)$ & $\begin{array}{l}0.889 \\
\quad(0.192-4.114)\end{array}$ & 0.880 & $\begin{array}{l}1.202 \\
\quad(0.180-8.025)\end{array}$ & 0.849 \\
\hline Lumbosacral, $n(\%)$ & $12(9.68)$ & $6(46.15)$ & 1 & & 1 & \\
\hline \multicolumn{2}{|c|}{ Number of segments involved } & & & 0.215 & & 0.561 \\
\hline 1 level, $n$ (\%) & $3(2.42)$ & $0(0.00)$ & $\begin{array}{l}0.000 \\
\quad(0.000-0.000)\end{array}$ & 0.999 & $\begin{array}{l}0.000 \\
\quad(0.000-0.000)\end{array}$ & 0.999 \\
\hline 2 levels, $n(\%)$ & $95(76.61)$ & $8(61.54)$ & $\begin{array}{l}0.219 \\
\quad(0.048-0.995)\end{array}$ & 0.049 & $\begin{array}{l}0.338 \\
\quad(0.046-2.476)\end{array}$ & 0.286 \\
\hline 3 levels, $n(\%)$ & $18(14.52)$ & $1(7.69)$ & $\begin{array}{l}0.563 \\
\quad(0.093-3.391)\end{array}$ & 0.530 & $\begin{array}{l}1.108 \\
\quad(0.118-10.438)\end{array}$ & 0.928 \\
\hline$\geq 4$ levels, $n$ (\%) & $8(6.45)$ & $4(30.77)$ & 1 & & 1 & \\
\hline Surgical approach & & & & 0.993 & & 0.993 \\
\hline Anterior, $n(\%)$ & $3(2.42)$ & $0(0.00)$ & $\begin{array}{l}0.000 \\
\quad(0.000-0.000)\end{array}$ & 0.999 & $\begin{array}{l}0.000 \\
\quad(0.000-0.000)\end{array}$ & 0.999 \\
\hline Posterior, $n(\%)$ & $58(46.77)$ & $6(46.15)$ & $\begin{array}{l}0.931 \\
\quad(0.296-2.932)\end{array}$ & 0.903 & $\begin{array}{l}1.114 \\
\quad(0.178-6.955)\end{array}$ & 0.908 \\
\hline
\end{tabular}


Table 5 continued

\begin{tabular}{|c|c|c|c|c|c|c|}
\hline \multirow[t]{2}{*}{ Characteristics } & \multirow{2}{*}{$\begin{array}{l}\text { Wound healing } \\
\text { or bone fusion } \\
(n=124)\end{array}$} & \multirow{2}{*}{$\begin{array}{l}\text { Wound non- } \\
\text { healing or bone } \\
\text { non-fusion } \\
(n=13)\end{array}$} & \multicolumn{2}{|c|}{ Univariate analysis } & \multicolumn{2}{|c|}{ Multivariate analysis } \\
\hline & & & $\begin{array}{l}\text { Odds ratio }(95 \% \\
\text { CI) }\end{array}$ & $P$ value & $\begin{array}{l}\text { Odds ratio }(95 \% \\
\text { CI) }\end{array}$ & $P$ value \\
\hline $\begin{array}{l}\text { Posterior and } \\
\text { anterior, } n(\%)\end{array}$ & $63(50.81)$ & $7(53.85)$ & 1 & & 1 & \\
\hline $\begin{array}{l}\text { Operation time } \\
(\min )\end{array}$ & $225.53 \pm 55.24$ & $236.46 \pm 71.62$ & $\begin{array}{l}1.003 \\
(0.993-1.013)\end{array}$ & 0.509 & $\begin{array}{l}1.005 \\
\quad(0.988-1.023)\end{array}$ & 0.570 \\
\hline Blood loss (ml) & $615.92 \pm 331.46$ & $650.00 \pm 394.76$ & $\begin{array}{l}1.000 \\
(0.999-1.002)\end{array}$ & 0.728 & $\begin{array}{l}0.999 \\
\quad(0.996-1.002)\end{array}$ & 0.436 \\
\hline $\begin{array}{l}\text { Concomitant } \\
\text { pulmonary TB, } \\
n(\%)\end{array}$ & $9(7.26)$ & $2(15.38)$ & $\begin{array}{l}2.323 \\
\quad(0.445-12.125)\end{array}$ & 0.317 & & \\
\hline
\end{tabular}

The indications for surgical intervention were neurological impairment, spinal instability, severe kyphotic deformity (more than $30^{\circ}$ ), and unresponsiveness to medical therapy

\section{Adverse Events}

Four and two patients in the 2- and 4-week group, respectively, needed prolonged percutaneous drainage of abscess. There was one patient who developed disseminated TB due to discontinued anti-TB therapy 8 months after operation by himself, while one other case had persistent preoperative pain in the incision region in the 4-week group. Another patient had malposition of screw in the 2-week group. There was no recurrence in either group during the 18 months' follow-up after completion of anti-TB therapy.

\section{DISCUSSION}

As a result of the deficiency of feasible diagnosis techniques, patients with spinal $\mathrm{TB}$ are more likely to be diagnosed late and with severe complications such as spinal deformities and neurological involvement, especially in resource-limited settings. In previous studies, the rate of surgical intervention among patients with spinal TB was reported to be $70 \%$ in India [11] and $77 \%$ in China [12], compared with only $20 \%$ in the USA [13]. Although the preoperative administration of anti-TB drugs is valued, the duration of the chemotherapy remains debatable [10, 14-16]. Two main factors should be considered to determine the right time for operation: (1) affirmation of anti-TB chemotherapeutic effects and (2) minimization of the delay in operation for patients with neurological deficits caused by spinal TB. According to several retrospective studies, the preoperative ATT durations were highly variable, ranging from 1 day to a few months $[10,14-16]$. Furthermore, there is a lack of knowledge regarding the appropriate preoperative duration of chemotherapy, and the given information was vague in almost all of the studies on the surgical treatment of vertebral tuberculosis. Although 2-4 weeks of chemotherapy is mostly advocated in clinical practice in China, there is no rationale behind this. Notably, patients at this stage suffer a lot from the disease including insomnia due to pain and limitation in movement that makes them need assistance from others routinely. An immediate operation, followed by immediate rehabilitation, could save them from these agonies and is the key to avoid sequela or movement dysfunction. 
In this study, we found that 2 and 4 weeks of preoperative anti-TB treatment produced similar surgical outcomes for spinal TB treatment. There was no significant difference between the two groups considering relapse. Neither group had any recurrences. This outcome might directly relate to the more than 18 months of chemotherapy in total. The Chinese Antituberculosis Association recommends a 12-18month treatment course for patients with spinal TB. In our clinical practice, an 18-month regimen is routinely applied because of the severity of spinal TB and because of the possibility of disease recurrence. This limitation made the long-term prognosis evaluation impossible in this study.

In this study, we only compared 2-week and 4-week preoperative ATT. Although further shortening of the preoperative ATT to 1 week and the impact of no preoperative anti-TB treatment are intriguing possibilities, further studies are needed in the near future to address them. Minimizing the waiting time for operation not only shortens the suffering time of the patient but also decreases the cost associated with hospitalization, and also enables earlier recovery of movement. However, in this study, several indicators were less sound in the 2-week group than in the 4-week group, although most of them were not significantly different, and none of them had significant influence on the key operation outcome indexes. The culture positive rate with pus in the 4 -week preoperative ATT group (41.94\%) was significantly lower than that in the 2-week group (60.94\%, $P=0.033)$, which means that longer preoperative antiTB treatment reduced bacilli in lesions more evidently. Nevertheless, the positive rate of Xpert assay with pus (86.21\%) was higher in the 4-week group than in the 2-week group $(80.00 \%)$, but the difference was not significant $(P=0.388)$. These inconsistent outcomes between culture and Xpert assay may reflect the less sensitive nature of culture, or reflect the inability of Xpert in differentiating dead or alive bacilli. Wound healing rate within 3 months after the operation $(94.20 \%, 65 / 69$ vs $89.71 \%$, $61 / 68 ; P=0.333$ ), bony fusion rate within 6 months $(98.46 \%, 64 / 65$ vs $95.45 \%, 63 / 66$; $P=0.317)$, and ESR normalization rate were higher in the 4-week preoperative ATT group than in the 2-week group. On the basis of these conditions, a well-designed study concerning even shorter preoperative ATT is needed.

In this study, the incidence of non-healing wound or non-fusion bone in thoracolumbar and lumbosacral spinal TB was significantly higher than that of other types of spinal TB. However, the two indexes were not significantly correlated with the number of vertebrae involved, surgical approach, and pus culture results. Similarly, Ren et al. [8] reported that lumbosacral and thoracolumbar TB had higher postoperative relapse and non-healing rates. This outcome may relate to the special anatomic and biomechanical characteristics of the spine. Theoretically, successful exposure of the junctional zones in the operation, such as the lumbosacral and thoracolumbar segment, is relatively difficult, which made the complete removal of the lesion from this location challenging.

\section{CONCLUSIONS}

Patients with spinal TB administered 2 or 4 weeks of preoperative antiTB treatment acquired similar surgical outcomes. Patients that received the operation sooner suffered 2 weeks' less agony from the disease. Shortening preoperative antiTB treatment duration time to less than 2 weeks is worthy of study.

\section{ACKNOWLEDGEMENTS}

We thank the participants of the study.

Funding. This work was supported by the research funding from Key Project of Department of Science and Technology Beijing (D141107005214002); Beijing Talents foundation (2018000021223), Beijing Municipal Administration of Hospitals' Ascent Plan (DFL20181602), and Beijing Municipal Administration of Hospitals Clinical Medicine Development of Special Funding Support 
(ZYLX201809). The journal's Rapid Service Fee was funded by the authors.

Authorship. All authors meet the International Committee of Medical Journal Editors (ICMJE) criteria for authorship for this article, take responsibility for the integrity of the work as a whole, and have given their approval for this version to be published.

Authors' Contributions. JF, TL, KT, and GW performed the data analysis and interpretation, drafted and revised the manuscript. WD, DL, GY, YL, and GL were responsible for doing the test. JS, QW, and JM were involved in recruiting patients. HH and SQ conceptualized the study, supervised the research design, data analysis, and interpretation; and critically reviewed and revised the manuscript. All authors read and approved the final manuscript.

Disclosures. Jun Fan, Tinglong Lan, Kai Tang, Guirong Wang, Weijie Dong, Dawei Li, Guangxuan Yan, Yuan Li, Guohua Lei, Jianlin Shan, Qinglei Wang, Jianhua Ma, Hairong Huang, and Shibing Qin declare that they have no conflict of interest.

Compliance with Ethics Guidelines. The ethical approvals for this study were obtained from the Medical Research Ethics Committee, Beijing Chest Hospital, Capital Medical University (BJXK-20150901) and ethics approvals were also obtained for all study sites (Table 1 in the supplementary material). The study was conducted according to the Helsinki Declaration of 1964, as revised in 2013, concerning human and animal rights, and Springer's policy concerning informed consent has been followed. Written informed consent was acquired from each participant.

Data Availability. The datasets generated during and analyzed during the current study are available from the corresponding author on reasonable request.

Open Access. This article is licensed under a Creative Commons Attribution-NonCommercial 4.0 International License, which permits any non-commercial use, sharing, adaptation, distribution and reproduction in any medium or format, as long as you give appropriate credit to the original author(s) and the source, provide a link to the Creative Commons licence, and indicate if changes were made. The images or other third party material in this article are included in the article's Creative Commons licence, unless indicated otherwise in a credit line to the material. If material is not included in the article's Creative Commons licence and your intended use is not permitted by statutory regulation or exceeds the permitted use, you will need to obtain permission directly from the copyright holder. To view a copy of this licence, visit http://creativecommons.org/licenses/by$\mathrm{nc} / 4.0 /$.

\section{REFERENCES}

1. Najafi A, Mahdian N, Yousefi B, et al. New vaccine candidates as a scientific solution against the dream of tuberculosis vaccine. Rev Med Microbiol. 2020;31(3):126-34.

2. Eslami M, Shafiei M, Ghasemian A, et al. Mycobacterium avium paratuberculosis and Mycobacterium avium complex and related subspecies as causative agents of zoonotic and occupational diseases. J Cell Physiol. 2019;234(8):12415-21.

3. Chipeio ML, Sayah A, Hunter CJ. Spinal tuberculosis. Am J Trop Med Hyg. 2021. https://doi.org/10. 4269/ajtmh.20-1529.

4. Boachie-Adjei O, Papadopoulos EC, Pellise F, et al. Late treatment of tuberculosis-associated kyphosis: literature review and experience from a SRS-GOP site. Eur Spine J. 2013;22(Suppl 4):641-6.

5. Li W, Liu Z, Xiao X, et al. Early surgical intervention for active thoracic spinal tuberculosis patients with paraparesis and paraplegia. BMC Musculoskelet Disord. 2021;22(1):213. https://doi.org/10.1186/ s12891-021-04078-y.

6. Shi JD, Wang ZL, Geng GQ, et al. Intervertebral focal surgery for the treatment of non-contiguous multifocal spinal tuberculosis. Int Orthop. 2012;36(7):1423-7.

7. Wang X, Pang X, Wu P, et al. One-stage anterior debridement, bone grafting and posterior instrumentation vs single posterior debridement, bone grafting, and instrumentation for the treatment of 
thoracic and lumbar spinal tuberculosis. Eur Spine J. 2014;23(4):830-7.

8. Ren HL, Jiang JM, Wang JX, et al. Is duration of preoperative anti-tuberculosis treatment a risk factor for postoperative relapse or non-healing of spinal tuberculosis? Eur Spine J. 2016;25(12): 3875-83.

9. Xu Z, Wang X, Liu Z. One-stage posterior debridement and single-segment interbody fusion for treating mono-segmental lumbar and lumbosacral spinal tuberculosis in adults following minimum 5-year follow-up. J Orthop Surg Res. 2020;15(1): 473. https://doi.org/10.1186/s13018-020-02005-w.

10. Yang L, Liu Z. Analysis and therapeutic schedule of the postoperative recurrence of bone tuberculosis. J Orthop Surg Res. 2013;8:47.

11. Patankar AP. Tuberculosis of spine: an experience of 30 cases over two years. Asian J Neurosurg. 2016;11(3):226-31.
12. Wu W, Lyu J, Liu X, et al. Surgical treatment of thoracic spinal tuberculosis: a multicenter retrospective study. World Neurosurg. 2018;110: e842-50.

13. De la Garza Ramos R, Goodwin CR, Abu-Bonsrah N, et al. The epidemiology of spinal tuberculosis in the United States: an analysis of 2002-2011 data. J Neurosurg Spine. 2017;26(4):507-12.

14. Dai LY, Jiang LS, Wang YR, et al. Chemotherapy in anterior instrumentation for spinal tuberculosis: highlighting a 9-month three-drug regimen. World Neurosurg. 2010;73(5):560-4.

15. Guzey FK, Emel E, Bas NS, et al. Thoracic and lumbar tuberculous spondylitis treated by posterior debridement, graft placement, and instrumentation: a retrospective analysis in 19 cases. J Neurosurg Spine. 2005;3(6):450-8.

16. Ling T, Liu L, Yang X, et al. Revision surgery for spinal tuberculosis with secondary deformity after treatment with debridement, instrumentation, and fusion. Eur Spine J. 2015;24(3):577-85. 